\title{
Transverse second-harmonic generation from disordered nonlinear crystals*
}

Research Article

\author{
Robert Fischer ${ }^{1}$, Solomon M. Saltiel ${ }^{13}$, Dragomir N. Neshev ${ }^{1}$, Wieslaw Krolikowski ${ }^{2 \dagger}$, Yuri S. Kivshar ${ }^{1}$ \\ 1 Nonlinear Physics Centre and Centre for Ultrahigh-bandwidth Devices for Optical Systems (CUDOS), Research School of \\ Physical Sciences and Engineering Australian National University, Canberra, ACT 0200, Australia \\ 2 Laser Physics Centre and Centre for Ultrahigh-bandwidth Devices for Optical Systems (CUDOS), Research School of \\ Physical Sciences and Engineering Australian National University, Canberra, ACT 0200, Australia \\ 3 Department of Quantum Electronics, Faculty of Physics, Sofia University, 5 J. Bourchier boulevard, BG-1164, Sofia, Bulgaria
}

Received 13 November 2007; accepted 19 March 2008

\begin{tabular}{|c|c|}
\hline Abstract: & $\begin{array}{l}\text { We investigate the transverse second-harmonic generation in as grown strontium barium niobate (SBN) } \\
\text { crystals with a random structure of anti-parallel ferroelectric domains. We consider both, single and } \\
\text { counter-propagating pulse geometries. We investigate polarization properties of the second harmonic } \\
\text { signal and discuss applications of this process for short pulses characterization. }\end{array}$ \\
\hline \multicolumn{2}{|l|}{ PACS (2008): 4} \\
\hline Keywords: & $\begin{array}{l}\text { second-harmonic generation } \cdot \text { disordered nonlinear media } \cdot \text { pulse duration measurement } \cdot \text { nonlinear } \\
\text { scattering }\end{array}$ \\
\hline & () Versita Warsaw and Springer-Verlag Berlin Heidelberg. \\
\hline
\end{tabular}

\section{Introduction}

Transverse second harmonic (TSH) generation by a single or two counter-propagating waves $[1,2]$ is a very challenging nonlinear optical effect. It can not be observed in bulk nonlinear crystals due to the lack of phase matching. In order to fulfill the required phase-matching condition, often layered or periodically poled media are used. The first successful experiments with TSH have been conducted in 1-2 $\mu \mathrm{m}$ thick quantum wells waveguide

*Presented at 9-th International Workshop on Nonlinear Optics Applications, NOA 2007, May 17-20, 2007, Świnoujście, Poland

†E-mail: wzk111@rsphysse.anu.edu.au structures [3-7]. The emission of TSH from these waveguides is frequently termed as the surface emitting second harmonic generation. As all these experiments used the counter-propagating short pulses the spatial distribution of the TSH represents the autocorrelation of the pulses and hence can be used for pulse duration monitoring $[3,4]$.

Recently we have shown that interaction of counterpropagating beams in the nonlinear quadratic crystal such as as grown strontium barium niobate (SBN) leads to TSH which can be used for convenient autocorrelation measurements of femtosecond pulses [8]. In this work we discuss in detail the physics of this transverse second harmonic generation process. We consider both, single and two counter-propagating pump beams geometries and analyze the relevant phase matching conditions. We will also dis- 
cuss polarization properties of this effect and show how they can be used to determine values of the nonzero components of the second order nonlinearity tensor.

\section{Single pump beam experiment}
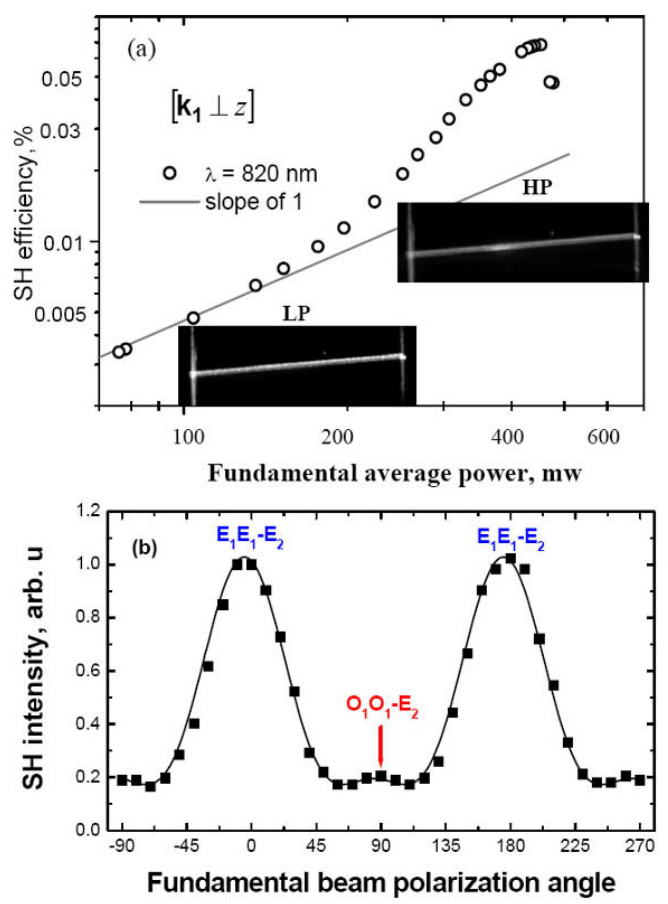

Figure 1. Single beam second harmonic generation in SBN crystal. (a) Strength of the second harmonic signal as a function of input power. Line represents the expected linear dependence between the second harmonic efficiency and input fundamental power. Insets show the photos from the top of the crystal of TSH radiation for $E_{1} E_{1}-E_{2}$ process, Axis $\mathrm{Z}$ is horizontal. HP - high fundamental power; LP - low fundamental power; b) dependence of the TSH signal on input polarization angle. $0^{\circ}$ and $180^{\circ}$ correspond to pure $E_{1} E_{1}-E_{2}$ process, while the points $190^{\circ} ; 90^{\circ}$ and $270^{\circ}$ correspond to single $\mathrm{O}_{1} \mathrm{O}_{1}-\mathrm{E}_{2}$ process. Line theoretical prediction with formula (2). Single fitting parameter is used - the maximum intensity for the $E_{1} E_{1}-E_{2}$ process.

In the experiments we used a femtosecond Ti:Sapphire oscillator (Mira, Coherent) emitting 150-170 fs (FWHM) pulses of $6-7 \mathrm{~nJ}$ energy at the repetition rate of $76 \mathrm{MHz}$, tunable in the range of $700-900 \mathrm{~nm}$. The laser beam (with an average power up to $550 \mathrm{~mW}$ ) is focused inside a $5 \times 5 \times 10 \mathrm{~mm}$ as-grown unpoled SBN:60 crystal. All sides of the SBN crystal have been polished allowing the observation of the second harmonic radiation from different directions. The properties of the fundamental and second harmonic pulses are characterized using a spec- trometer (HR2000, Ocean Optics) and a GRENOUILLE system (UPM 8-50, Newport). The estimated peak power of a single pulse is less than the critical power for selffocusing due to electronic (instantaneous) contribution of the $\chi^{(3)}$ nonlinearity of the SBN crystal. At low average input powers the divergence of the fundamental beam after its propagation in the SBN crystal is defined by the focal spot ( 6.5 mrad @ $100 \mathrm{~mW})$, whereas its spectral width is inversely proportional to the pulse duration.

To measure the efficiency of a single beam TSH the crystal is illuminated perpendicularly to its optical Z-axis. In this particular measurement we explored the interaction $E_{1} E_{1}-E_{2}$, where both fundamental beam $\left(E_{1}\right)$ and second-harmonic signal $\left(E_{2}\right)$ are extraordinary polarized and hence is governed by the strongest second order nonlinear component $d_{33}$. The efficiency for the single beam TSH generation for this geometry of excitation as a function of input power is shown in Fig. 1. The quadratic (instead of expected linear) increase of the TSH signal above $250 \mathrm{~mW}$ average power is the result of a two-photon absorption induced thermal lensing in the crystal. The insets in Fig. 1 show the spatial structure of the transversely emitted second harmonic and demonstrate the effect of thermal self-focusing at high power. The maximal efficiency shown in Fig. 1(a) of about $0.1 \%$ corresponds to emission from a single direction (surface). Taking into account the emission in opposite directions the total efficiency of TSH approaches $0.2 \%$.

The dependence of the TSH signal on the input polarization is shown in Fig. 1(b). Its complex character clearly indicates that two processes are involved in TSH scattering: $E_{1} E_{1}-E_{2}$ and $O_{1} O_{1}-E_{2}$. In the second process two ordinary fundamental waves $\left(\mathrm{O}_{1}\right)$ generate extraordinary second harmonic wave $\left(E_{2}\right)$. The maximal intensity points (at $0^{\circ}$ and $180^{\circ}$ of the input polarization angle) correspond to pure $E_{1} E_{1}-E_{2}$ process, while the points $-90^{\circ} ; 90^{\circ}$ and $270^{\circ}$ correspond to the $\mathrm{O}_{1} \mathrm{O}_{1}-\mathrm{E}_{2}$ process. For intermediate polarization both processes contribute toward the generation of second harmonic signal. It is worth stressing that the simultaneous phase matching of two SHG processes $E_{1} E_{1}-E_{2}$ and $O_{1} O_{1}-E_{2}$ is only possible thanks to the random domain distribution in the crystal. Typically such simultaneous phase matching requires special techniques [9]. However, in this untreated (as-grown) SBN crystal we get it "for free". The $\mathrm{O}_{1} \mathrm{O}_{1}-\mathrm{E}_{2}$ and $\mathrm{E}_{1} \mathrm{E}_{1}-\mathrm{E}_{2}$ processes are governed by the $d_{32}$ and $d_{33}$ nonlinear coefficients, respectively. Therefore, as will be shown later (section 4), the dependence of the second harmonic signal on the input polarization can be used to extract the information about the ratio of these different nonlinear coefficients. 


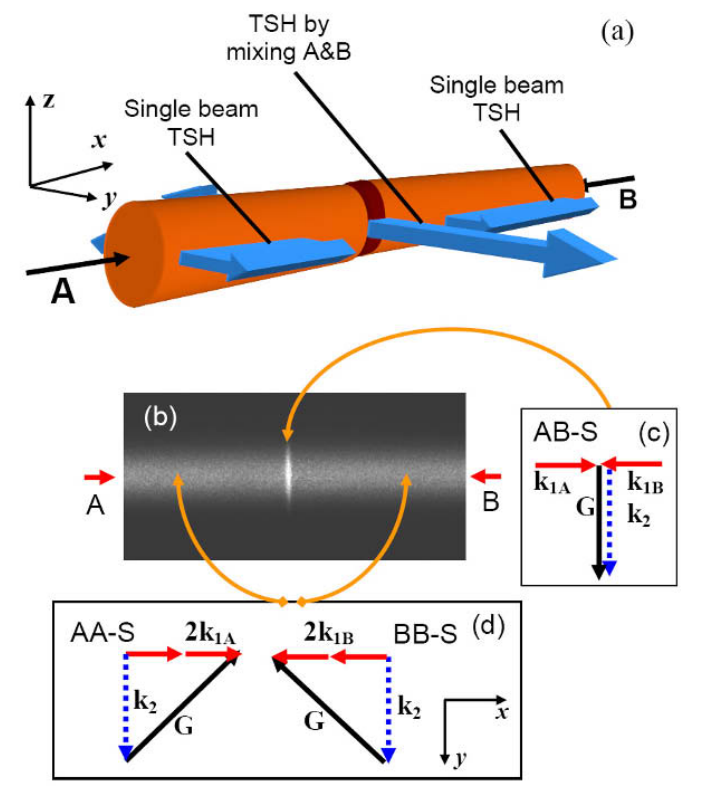

Figure 2. (a) Schematic sketch of the concept of counterpropagating TSH generation. (b) The second harmonic signal recorded by the CCD camera. The phase matching conditions for second harmonic emission with counter-propagating (c) and individual (d) pulses. G represents the reciprocal grating of the random structure that ensures the fulfillment of the phase matching condition.

\section{Experiments with counter- propagating pulses}

The interaction scheme with counter-propagating fundamental beams $A$ and $B$ is illustrated in Fig. 2(a). The optical paths of both beams are chosen such that the pulses meet in the middle of the crystal (Fig. 3 top). There are now three different contributions to the overall TSH signal. The first two come from the TSH generation by each individual pulse. They are depicted in Fig. 2(a) by wide arrows. This emission takes place along the whole crystal. The third contribution is due to a two-pulse process and is emitted only from the area where both pulses overlap. A typical trace of the TSH recorded by a CCD camera mounted perpendicularly to the beams is shown in Fig. 2(b). The phase matching conditions for single and two-pulse processes are shown in Fig. 2(c,d). G represents the random structure gratings that enables phase matching. In periodically poled nonlinear crystals $\mathrm{G}=$ $m(2 \pi / \Lambda)$, with $\wedge$ the period of modulation of second order nonlinearity and $\mathrm{m}$ - integer. Since domain structure of our SBN crystal is random and the sizes of the domains vary randomly from 0.5 to $20 \mu \mathrm{m}$ the vectors $\mathrm{G}$ can take any value in a range defined by this distribution of sizes
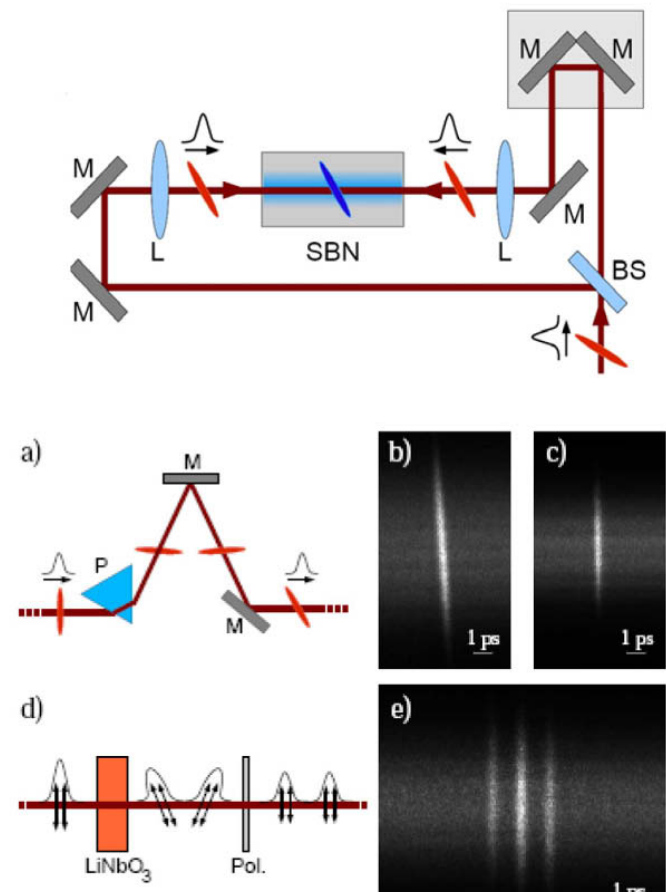

e)

Figure 3. Detection of pulse front tilt and pulse trains with TSH generation in SBN crystal. Top: Set-up of the autocorrelator. (a) Optical system used to induce a pulse front tilt; (b-c) autocorrelation traces of the tilted and straight front pulses. (d) Schematic of generation of a pulse doublet by means of a birefringent crystal. (e) The autocorrelation trace of the doublet for 990 fs delay between the constituent pulses.

of the domains.

The emitted $\mathrm{SH}$ was measured to be always extraordinary polarized independently of the input polarization of the beams $A$ and $B$, However, the intensity of the TSH signal was very sensitive to the input polarizations of both beams $A$ and $B$.

In this particular configuration, when the two counterpropagating pulses are identical, the generated normalized signal $I_{\text {tot }}(\tau)$ is given as $I_{\text {tot }}(\tau)=1+\frac{2}{Q} G^{(2)}(\tau)$ (see e.g. [10]) where $G^{(2)}(\tau)=\langle I(t) I(t+\tau)\rangle /\left\langle I^{2}(t)\right\rangle$ represents exactly the autocorrelation of the pulse and $Q$ is the ratio of the efficiencies of both processes: single and counter-propagating beams TSH. This experimental geometry results in a mapping of time $(\tau)$ into a space (longitudinal) coordinate $(x)$ via the simple relation $\tau=(x-$ $\left.x_{0}\right) 2 n_{\omega} / c$, where $n_{\omega}$ is the SBN refractive index dependent on the optical frequency, $c$ is the speed of light in vacuum and $x_{o}$ is the center of the overlapping area. Hence, the longitudinal distribution of the second harmonic signal represents a time-dependent autocorrelation signal. We 


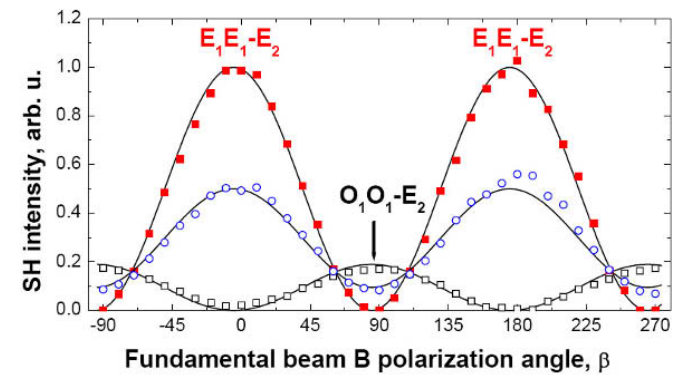

Figure 4. Transverse second harmonic with counter-propagating beams. The experimental dependences of the second harmonic signal on input polarization angle $\beta$ of beam $\mathrm{B}$, for three fixed polarization angle $(\alpha)$ of the beam $\mathrm{A}$ : extraordinary, $\alpha=0$, (full squares), intermediate, $\alpha=45^{\circ}$ (open circles) and ordinary, $\alpha=90^{\circ}$ (open squares); lines - theoretical predictions with formula (3). Single fitting parameter is used - the maximum intensity for the $E_{1} E_{1}-E_{2}$ process.

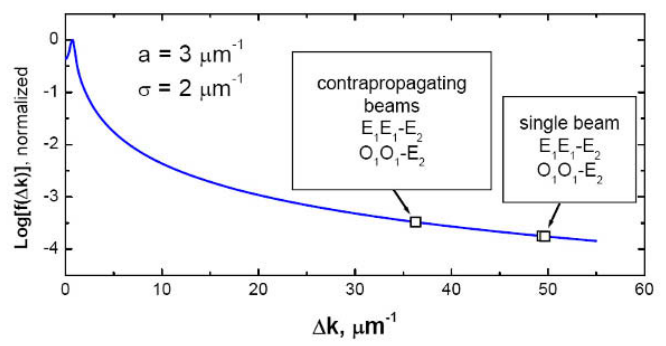

Figure 5. Function $f(\Delta k)$ plotted for the case of $a=3 \mu \mathrm{m}$ and $\sigma=2 \mu \mathrm{m}$. By open squares we denote the values corresponding to $E_{1} E_{1}-E_{2}$ and $O_{1} O_{1}-E_{2}$ second harmonic generation processes.

note that this is a single-shot technique that unlike traditional autocorrelators does not require any variation of a delay between interacting pulses. Measurements with a CCD camera were done at the power levels down to $0.26 \mathrm{MW} / \mathrm{cm}^{2}$, four orders of magnitude lower than those usually used with the two photon fluorescence (TPF) technique $[10,11]$. This significantly lower power requirement is mainly due to the confinement of the emitted $\mathrm{SH}$ in a plane compared to the emission in a full three dimensional solid angle of the TPF process.

Apart from the pulse duration measurements, the TSH arising from mixing both pulses can be also used to visualize unambiguously the tilt of the front of an optical pulse. Such a pulse front tilt (PFT) which is typically caused by dispersive elements like prisms, gratings, or wedges leads to effectively longer pulse duration and hence a lower peak power in the focal plane of the beam. To demonstrate the ability for detection of PFT we placed a $60^{\circ}$ (SF11 Schott

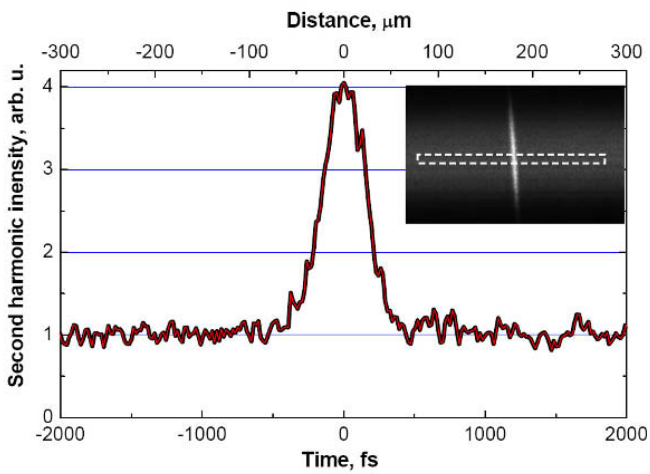

Figure 6. Autocorrelation trace obtained with TSH in SBN with two counter-propagating pulses. The values of all data points are normalized to the background value. The peak/background ratio is 4 .

glass) prism into beam path (Fig. 3(a)) inducing a $4^{\circ}$ pulse front tilt inside the crystal (Fig. 3(b)). The angular tilt of the trace compared to the trace without the prism (Fig. $3(c))$ is a clear indication of the presence of PFT.

Unlike the standard techniques which rely on measurements conducted within a narrow time window and assume an identical form for all pulses in a pulse train, our technique can be used in measurements over a large time window. Therefore it can properly decode temporal structures consisting of multiple pulses. To demonstrate this feature we prepared a signal beam in form of a series of two closely propagating pulses. These pulses are created from the original pulse train by passing it through a thin $(3 \mathrm{~mm})$ sample of a birefringent (lithium niobate) crystal followed by a polarizer, as depicted in Fig. 3(d). In this way, the input pulse is split into a sequence of two pulses with their temporal spacing determined by the birefringence and thickness of the crystal. Here the delay between pulses was roughly $1 \mathrm{ps}$. The autocorrelation trace shown in Fig. 3(e) clearly resolves the two components of a pulse doublet allowing for a delay between them to be precisely measured. For comparison, in the analogous experiment with GRENOUILLE the two-pulse character of the signal could not be resolved because of the small temporal window inherent to its design.

Optimization of the process of TSH by mixing of two beams requires knowledge of the dependence of its intensity on the polarization of the input beams. Results of the polarization measurements together with theoretical predictions are depicted in Fig. 4. The plots show the dependence of the autocorrelation signal (with background removed) as the function of the input polarization angle of the beam $B$ for three different polarization angles $(\alpha)$ 
of the beam $A$. The largest emission efficiency is again obtained for the case when both beams are extraordinary polarized and the second harmonic generation is due to the $E_{1} E_{1}-E_{2}$ process. The lowest generation efficiency is for the process $\mathrm{O}_{1} \mathrm{O}_{1}-\mathrm{E}_{2}$ when both input beams are ordinary polarized. In the case when the input polarization of the beam $A$ is oriented at $45^{\circ}$ angle, the generation of the second harmonic wave is due to two simultaneously acting processes: $\mathrm{E}_{1} \mathrm{E}_{1}-\mathrm{E}_{2}$ and $\mathrm{O}_{1} \mathrm{O}_{1}-\mathrm{E}_{2}$.

\section{Theoretical model and discus- sions}

In the following theoretical analysis of the TSH generation in the random system we will use the one-dimensional approach of Le Grand et al [12]. While, strictly speaking, the case considered here is two-dimensional, it turns out that even the simple 1D theory already provides a fairly good agreement with the experimental results. Following [12] and the discussions by Morozov and Chirkin [13] and Dolino [14] one can show that the intensity of the $\mathrm{SH}$ wave generated in a medium with random distribution of ferroelectric domains is given by

$$
l_{2} \propto \frac{2 n_{2 \omega}}{\varepsilon_{o} c n_{\omega}^{2}}\left(\frac{\omega}{c n_{2 \omega}}\right)^{2} d_{e f f}^{2} l_{1}^{2} L,
$$

where $L$ denotes the interaction length, the coefficient $d_{\text {eff }}^{2}$ depends on the type of the interaction $\left(E_{1} E_{1}-E_{2}\right.$ or $\mathrm{O}_{1} \mathrm{O}_{1}$ $\mathrm{E}_{2}$ ), bulk wave vector mismatch $\Delta k$ and statistical properties of the domain distribution. As a matter of fact, the statistical character of the second harmonic generation process is already evident in the linear (unlike the usual quadratic) dependence of $\mathrm{I}_{2}$ on the interaction length [15]. The explicit form of the expressions for $d_{e f f}^{2}$ is given as

$$
d_{e f f, s b}^{2}=d_{33}^{2} f\left(\Delta k_{e e-e}^{(s b)}\right) \cos ^{4} \beta+d_{32}^{2} f\left(\Delta k_{o o-e}^{(s b)}\right) \sin ^{4} \beta,
$$

for single beam TSH generation and

$$
\begin{aligned}
d_{e f f, c p}^{2} & =4 d_{33}^{2} f\left(\Delta k_{e e-e}^{(c p)}\right) \cos ^{2} \beta \cos ^{2} \alpha \\
& +4 d_{32}^{2} f\left(\Delta k_{o o-e}^{(c p)}\right) \sin ^{2} \beta \sin ^{2} \alpha
\end{aligned}
$$

for counter-propagating TSH generation (propagation direction perpendicular to the $Z$-axis). The angles $\beta$ and $\alpha$ are measured from the $Z$ axis. This means, for example, that for $\beta=0$ and $\alpha=0$ both beams are extraordinary polarized.
In derivation of Eqs. (2)-(3) we assumed no interference between the second harmonics signals from the two processes $E_{1} E_{1}-E_{2}$ and $\mathrm{O}_{1} \mathrm{O}_{1}-\mathrm{E}_{2}$ which is justified by the statistical nature of the whole second harmonic emission. The function $f(\Delta k, a, \sigma)$ is determined by the statistics of domain distribution. It is given as [12]

$$
\begin{aligned}
& f(\Delta k, a, \sigma) \propto \frac{4}{\Delta k^{2}} \\
& \times \frac{1-\exp \left(-\sigma^{2} \Delta k^{2}\right)}{1+\exp \left(-\sigma^{2} \Delta k^{2}\right)+2 \cos (a \Delta k) \exp \left(-\sigma^{2} \Delta k^{2} / 2\right)},
\end{aligned}
$$

where a and $\sigma$ denote mean size of the domains and dispersion of the size distribution, respectively.

The relative strength of the two processes $E_{1} E_{1}-E_{2}$ and $\mathrm{O}_{1} \mathrm{O}_{1}-\mathrm{E}_{2}$, as seen from Eqs. (2)-(3), depends on the coefficient

$$
R=\frac{I_{o o-e}}{l_{e e-e}}=\frac{f\left(\Delta k_{o o-e}^{(s b)}\right)}{f\left(\Delta k_{e e-e}^{(s b)}\right)}\left(\frac{d_{32}}{d_{33}}\right)^{2} .
$$

Hence, it is possible to extract the information about $\left|d_{32} / d_{33}\right|$ in case the function $f(\Delta k, a, \sigma)$ is known. From the published data on the domain size distributions in unpoled SBN crystal $[16,17]$ we estimated $a=3 \mu \mathrm{m}$ and $\sigma=2 \mu \mathrm{m}$. Then the corresponding plot of the function $f(\Delta k, a, \sigma)$ is shown in Fig. 5. The bulk phase mismatch $\Delta k$ of the processes $\mathrm{E}_{1} \mathrm{E}_{1}-\mathrm{E}_{2}$ and $\mathrm{O}_{1} \mathrm{O}_{1}-\mathrm{E}_{2}$ depends on the interaction geometry. For single beam TSH $\Delta k_{o o-e}^{(s b)}=$ $\sqrt{k_{2 e}^{2}+4 k_{1 o}^{2}}=49.7 \mu m^{-1} ; \Delta k_{e e-e}^{(s b)}=\sqrt{k_{2 e}^{2}+4 k_{1 e}^{2}}=$ $49.4 \mu \mathrm{m}^{-1}$ while for the TSH via counter-propagating pulses $\Delta k_{o o-e}^{(c p)}=\Delta k_{e e-e}^{(c p)}=k_{2 e}=36.3 \mu m^{-1}$. From this data we obtain $f\left(\Delta k_{o o-e}^{(c p)}\right)=f\left(\Delta k_{e e-e}^{(c p)}\right)=f\left(k_{2 e}\right)=$ $1.5 \times 10^{-4}$ and $f\left(\Delta k_{o o-e}^{(s b)}\right) \approx f\left(\Delta k_{e e-e}^{(s b)}\right)=0.8 \times 10^{-4}$ Consequently Eq. (5) leads to $R=\left(d_{32} / d_{33}\right)^{2}=0.19$, which gives $d_{32} / d_{33}=0.44$. This agrees very well with the value of 0.5 found experimentally in [18] and theoretically predicted value of 0.38 [19]. It is interesting to note that since $f\left(\Delta k_{o o-e}^{(s b)}\right) / f\left(\Delta k_{e e-e}^{c p}\right)=Q=0.54$, the signal to background contrast of the autocorrelation function is predicted to be 4.7 which exceeds the $3: 1$ ratio limitation of the two photon fluorescence technique [20]. Again, this is in a good agreement with experimentally determined value of 4 (see Fig. 6).

In conclusion, we discussed transverse second harmonic generation in SBN crystal with random distribution of the ferroelectric domains. We investigated the polarization properties of the process and measured the ratio of the two nonzero components of the second order nonlinearity $d_{32} / d_{33}$ in SBN crystals. Due to its naturally disordered structure, this crystal allows for simultaneous phase-matching of different processes, as demonstrated for 
the second harmonic generation from ordinary and extraordinary polarized fundamental beams. It is shown that the transverse second harmonic generation in crystals with random distribution of the ferroelectric domains can be used efficiently as a simple autocorrelator for the short pulse characterization.

\section{Acknowledgements}

The authors acknowledge the support of the Australian Research Council. S.S thanks the Nonlinear Physics Centre at the Australian National University for a warm hospitality during his stays in Canberra.

\section{References}

[1] X. Gu, R.Y. Korotkov, Y.J. Ding, J.U. Kang, J.B. Khurgin, J. Opt. Soc. Am. B 15, 1561 (1998)

[2] G.D. Landry, T.A. Maldonado, J. Opt. Soc. Am. B 21, 1509 (2004)

[3] R. Normandin, R.L. Williams, F. Chatenoud, Electr. Lett. 26, 2088 (1990)

[4] N.D. Whitbread, J.A.R. Williams, J.S. Roberts, I. Bennion, P.N. Robson, Opt. Lett. 19, 2089 (1994)

[5] A. Otomo et al., J. Opt. Soc. Am. B 15, 759 (1998)

[6] A. Fiore, et al., Appl. Phys. Lett. 70 , 2655 (1997)
[7] T.M. Crawford, C.T. Rogers ,T.J. Silva, Y.K. Kim, Appl. Phys. Lett. 68, 1573 (1996)

[8] R. Fischer et al., Appl. Phys. Lett. 91, 031104 (2007)

[9] S.M. Saltiel, A.A. Sukhorukov, Yu S Kivshar, Progress in Optics 47, 1 (2005)

[10] J.R. Klauder, M.A. Duguay, J.A. Giordmaine, S.L. Shapiro, Appl. Phys. Lett. 13, 174 (1968)

[11] J.A. Giordmaine, P.M. Rentzepis, S.L. Shapiro, K.W. Wecht, Appl. Phys. Lett. 11, 216 (1967)

[12] Y. Le Grand, D. Rouede, C. Odiu, R. Aubry, S. Mattauch, Opt. Commun. 200, 249 (2001)

[13] E. Yu Morozov, A.S. Chirkin, Quantum Electronics 34, 227 (2004)

[14] G. Dolino, Phys. Rev. B 6, 4025 (1972)

[15] M. Baudrier-Raybaut, R. Haïdar, Ph. Kupecek, Ph. Lemasson, E. Rosencher, Nature (London) 432, 374 (2004)

[16] J.J. Romero, C. Aragó, J.A. Gonzalo, D. Jaque, J.G. Solé, J. Appl. Phys. 93, 3111 (2003)

[17] M.O. Ramirez. D. Jaque, L. Ivleva, L.E. Bausa, J. Appl. Phys. 95, 6185 (2004)

[18] M. Horowitz, A. Bekker, B. Fischer, Appl. Phys. Lett. 62, 2619 (1993)

[19] S. Singh, In: R.J. Pressley (Ed.), Handbook of Lasers with Selected Data on Optical Technology, (The Chemical Rubber Co., Cleveland, 1971) Chap. 18

[20] J.H. Bechtel, W. Smith, J. Appl. Phys. 46, 5055 (1975) 